\title{
Fidelity of Analytic Drop Size Distributions in Drizzling Stratiform Clouds Based on Large-Eddy Simulations
}

\author{
YeFIM L. KOGAN AND ZENA N. KOGAN \\ Cooperative Institute for Mesoscale Meteorological Studies, University of Oklahoma, Norman, Oklahoma \\ DAVID B. MECHEM \\ Department of Geography, University of Kansas, Lawrence, Kansas
}

(Manuscript received 8 December 2008, in final form 30 January 2009)

\begin{abstract}
Cloud microphysical parameterizations and retrievals rely heavily on knowledge of the shape of drop size distributions (DSDs). Many investigations assume that DSDs in the entire or partial drop size range may be approximated by known analytical functions. The most frequently employed approximations of function are of the type of gamma, lognormal, Khrgian-Mazin, and Marshall-Palmer. At present, little is known about the accuracy of these approximations. The authors employ a DSD dataset generated by the Cooperative Institute for Mesoscale Meteorological Studies Large-Eddy Simulation (CIMMS LES) explicit microphysics model for stratocumulus cases observed during the Atlantic Stratocumulus Transition Experiment (ASTEX) field project. The fidelity of analytic lognormal- and gamma-type DSD functions is evaluated according to how well they represent the higher-order moments of the drop spectra, such as precipitation flux and radar reflectivity. It is concluded that for boundary layer marine drizzling stratocumuli, a DSD based on the twomode gamma distribution provides a more accurate estimate of precipitation flux and radar reflectivity than the DSD based on the lognormal distribution. The gamma distribution also provides a more accurate radar reflectivity field in two- and three-moment bulk microphysical models compared to the conventional $Z-R$ relationship.
\end{abstract}

\section{Introduction}

Many problems in the development of cloud parameterizations and remote sensing microphysical retrievals require knowledge of the drop size distribution (DSD). For example, accurate approximations of DSDs are needed to formulate expressions for the drop sedimentation process and microphysical growth rates from collision-coalescence. A reasonable DSD approximation is also important in obtaining a correct estimation of rain based on observed radar reflectivity (via $Z-R$ relationships). The approximation of DSDs by analytical fits provides a practical approach in representing DSDs in models that cannot resolve the full range of the drop size spectrum. Many of these bulk models formu-

Corresponding author address: Yefim L. Kogan, CIMMS, University of Oklahoma, 120 David L. Boren Blvd., Suite 2100, Norman, OK 73072-7304.

E-mail: ykogan@ou.edu late microphysical processes using one, two-or in more complex formulations-three moments of the DSD. These microphysical parameterizations assume that DSDs in either the whole or parts of the drop size range may be approximated by known analytical functions.

The most frequently employed approximations are gamma or lognormal functions; in more specific cases, the Khrgian-Mazin-type or Marshall-Palmer-type distributions are employed. For example, Feingold and Levin (1986), using observation of frontal convective clouds in Israel, showed that rain drop spectra can be approximated by the lognormal distribution. Willis (1984) analyzed DSDs from convective clouds in two hurricanes and found that a gamma distribution provided a best fit to the data. He suggested that results should apply to any convective clouds where the warm rain process applies through a deep vertical layer. Lognormal fits to forward scattering spectrometer probe (FSSP) spectra observed during the Atlantic stratocumulus transition experiment (ASTEX) yielded reasonable 
values of effective radius, with greater uncertainty associated with enhanced concentrations of drizzle droplets $(r>20 \mu \mathrm{m})$ (Gerber 1996). Cerro et al. (1997) found that the gamma distribution was generally able to represent rain drop distributions observed in precipitation episodes, with rain rates predominant in the range up to $5 \mathrm{~mm} \mathrm{~h}^{-1}$. Wood (2005) presented two examples of drizzle drop spectra fitted by an exponential function, which is a limiting case of the gamma distribution. In one example, the exponential fit gave an accurate estimation of precipitation rate and reflectivity, while in another example these parameters were overestimated. Both lognormal (Frisch et al. 1998) and gamma distributions (O'Connor et al. 2005) have been used in the development of algorithms for the retrieval of microphysical parameters. It is clear that no unanimity exists on the question of how best to represent the DSD in stratocumulus clouds.

At present, little is known about the accuracy of these approximations, especially their ability to successfully represent the higher-order moments of the DSD, such as reflectivity. Higher-order moments are difficult to estimate from measured drop spectra because large uncertainties exist in measurements of DSDs by optical array probes, especially near the tail of the spectra (see, e.g., Fig. 2 in Wood 2005). These uncertainties lead to large errors in estimates of rain rates and even greater errors in reflectivity. In this study, we evaluate the accuracy of lognormal- and gamma-type distributions in approximating higher-order moments of the DSDs. Our study is focused on drizzling stratocumulus clouds with predominantly bimodal distributions. We do not consider nonprecipitating clouds because cloud drop spectra in these cloud types are unimodal, and as we show below, their analytical approximation either by unimodal lognormal or gamma distribution can be quite accurate.

The advantage of large-eddy simulation (LES) model data is that the DSDs represent a simultaneous, threedimensional snapshot of a wide range of in-cloud microphysical conditions. In situ particle probe observations, on the other hand, are restricted to the one-dimensional path of the aircraft. Beyond this, however, both approaches have limitations. Even with careful experimental design, instrumental data can be wrought with uncertainty, such as in the case of optical probes where large sample sizes (long flight legs) are necessary to obtain statistically meaningful measurements of drops in the drizzle size range. In the model, uncertainties in the simulated DSDs can be attributed to the formulation of fundamental microphysical processes, subgridscale condensation and activation, and inhomogeneous mixing effects-any of which can influence the simulated DSDs.
Section 2 provides a brief description of the Cooperative Institute for Mesoscale Meteorological Studies (CIMMS) large-eddy simulation cloud model, the simulated cases, and the definition of analytical fits. In section 3, we present the evaluation of different analytical approximations. Results are discussed and summarized in section 4 .

\section{Approach}

\section{a. Model and data}

The basic features of the CIMMS LES cloud model (Khairoutdinov and Kogan 1999) are briefly summarized here. The thermodynamic state is described in terms of virtual liquid water potential temperature and total water mixing ratio. Cloud physics processes are formulated based on prediction equations for cloud condensation nuclei and cloud/drizzle drops (19 and 25 bins, respectively). A detailed description of the model can be found in Kogan (1991), Kogan et al. (1995), and Khairoutdinov and Kogan (1999). Individual case studies and comparison of simulations with aircraft observations (Khairoutdinov and Kogan 1999; Liu et al. 2000) have demonstrated that the model can reproduce the major dynamical, radiative, and microphysical parameters reasonably well. Indirect tests of a bulk drizzle parameterization derived from model-simulated spectra (Khairoutdinov and Kogan 1999) showed good agreement with a large number of observational datasets (Wood et al. 2002; Wood 2005).

For this study we simulated several cases of stratocumulus clouds observed during the ASTEX field experiment (Albrecht et al. 1995). The setup of the model and the initial thermodynamic profiles were similar to the profiles used in Khairoutdinov and Kogan (1999). The initial aerosol size distributions were based on the ASTEX A212 and A209 cases and transformed to activation spectra as detailed in Kogan (1991). Flight A212 was characterized by light drizzle, while the smaller aerosol concentrations in A209 were typical for stronger drizzle. The simulated cloud layers are drawn from three cases: a light drizzle (LD) case, based on aerosol conditions from flight A212, and moderate drizzle (MD) and heavy drizzle (HD) cases, based on two distinct air masses sampled during flight A209. These three labels (light, moderate, and heavy) represent different intensities of drizzle in the cloud. ${ }^{1}$ Each case provided more than 19000 DSDs collected from points inside the cloud with LWC larger than $0.01 \mathrm{~g} \mathrm{~m}^{-3}$. The range of cloud and drizzle parameters for all the performed simulations is

\footnotetext{
${ }^{1}$ Drizzle is defined as drops in the $25-300-\mu \mathrm{m}$ radius range.
} 
TABLE 1. Mean and standard deviation (in brackets) of drop spectra parameters for $\mathrm{LD}, \mathrm{MD}$, and HD cases. Here, $Q_{l}$ is liquid water content, $N_{c}$ is cloud drop concentration, $R_{m}$ is the mean radius of drop spectrum, $R$ is drizzle flux, and $Z_{d}$ is reflectivity.

\begin{tabular}{lccc}
\hline PARAMETER & LD & MD & HD \\
\hline$Q_{l}\left(\mathrm{~g} \mathrm{~m}^{-3}\right)$ & $0.32(0.14)$ & $0.30(0.16)$ & $0.26(0.16)$ \\
$R_{m}(\mu \mathrm{m})$ & $11.2(1.7)$ & $11.2(2.05)$ & $12.5(3.0)$ \\
$N_{c}\left(\mathrm{~cm}^{-3}\right)$ & $39.5(12.4)$ & $27.4(10.6)$ & $18.8(9.4)$ \\
$R\left(\mathrm{~mm} \mathrm{~d}^{-1}\right)$ & $0.84(0.45)$ & $1.7(0.98)$ & $2.2(1.3)$ \\
$Z_{d}(\mathrm{~dB} Z)$ & $-17.9(3.2)$ & $-9.8(4.8)$ & $-8.2(4.5)$ \\
\hline
\end{tabular}

shown in Table 1 and is illustrated in Fig. 1. The simulated cloud parameters are characteristic for drizzling stratocumuli evolving under clean maritime conditions.

Figure 2 shows examples of cloud drop spectra superimposed with vertical wind velocities in the moderate drizzle case simulation. Note the prevalence of bimodal cloud drop distributions.

\section{b. Definition of the analytical fits}

The DSD $n(r)$ is defined as the number concentration of particles in a size interval $r$ to $r+d r$ per unit volume. The total concentration (or the total number of particles per unit volume) $N$ is then

$$
N=\int_{r=0}^{\infty} n(r) d r
$$

Various moments of the DSD function define integral cloud microphysical parameters. For instance, the third moment is proportional to the liquid water content; the sixth moment defines the radar reflectivity in the Rayleigh limit. In boundary layer stratocumulus, the drizzle drop fall velocity can be reasonably well approximated by a linear function of drop radius. As a result, the precipitation flux in such clouds is proportional to the fourth moment of the drop distribution. With this in mind, it is important to be able to approximate cloud DSDs by an analytical fit, the moments of which can be integrated analytically. The most commonly employed approximations are general gamma or lognormal functions.

Gamma or lognormal distributions (Feingold and Levin 1986; Frisch et al. 1998) are defined by two parameters, which can be related to the mean radius and spectral width of the observed DSD. To obtain a specific spectrum, at least one integral parameter, such as the total particle concentration or liquid water content, must also be known. Therefore, a total of three parameters are required to describe a specific gamma or lognormal DSD.

The three-parameter lognormal fit (referred to as L-fit) is defined as
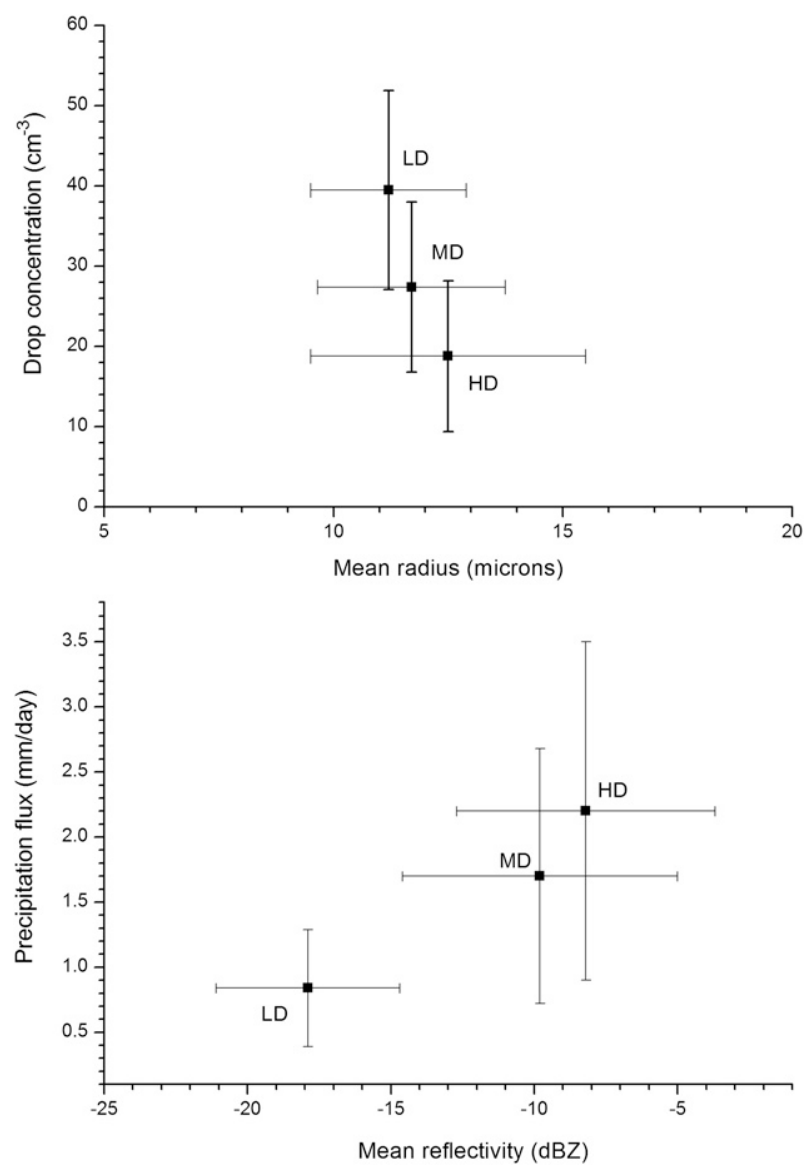

FIG. 1. Range of cloud parameters in the analyzed cases of stratocumulus cloud layers. The squares represent the means, and the error bars represent the standard deviation of a parameter.

$$
n(r)=\frac{N}{r \sigma_{\ln r} \sqrt{2 \pi}} \exp \left[-\frac{1}{2}\left(\frac{\ln r-\ln r_{m}}{\sigma_{\ln r}}\right)^{2}\right],
$$

where $N$ is the total concentration, $\sigma_{\ln r}$ is the logarithmic width of the distribution, and $r$ and $r_{m}$ are the radius and modal radius, respectively. The $k$ th moment of the distribution $M_{p}$ is given by

$$
M_{k}=\int_{r=0}^{\infty} r^{k} n(r) d r=N r_{m}^{k} \exp \left(\frac{k^{2} \sigma_{\ln r}^{2}}{2}\right) .
$$

For instance, the liquid water content $Q$ is defined as

$$
Q=\frac{4}{3} \pi \rho_{w} N r_{m}^{3} \exp \left(\frac{9 \sigma_{\ln r}^{2}}{2}\right) .
$$

The three-parameter gamma fit (referred to as G-fit) is defined as

$$
n(r)=\frac{N}{\Gamma(\alpha+1) \beta^{\alpha+1}} r^{\alpha} \exp \left(-\frac{r}{\beta}\right),
$$




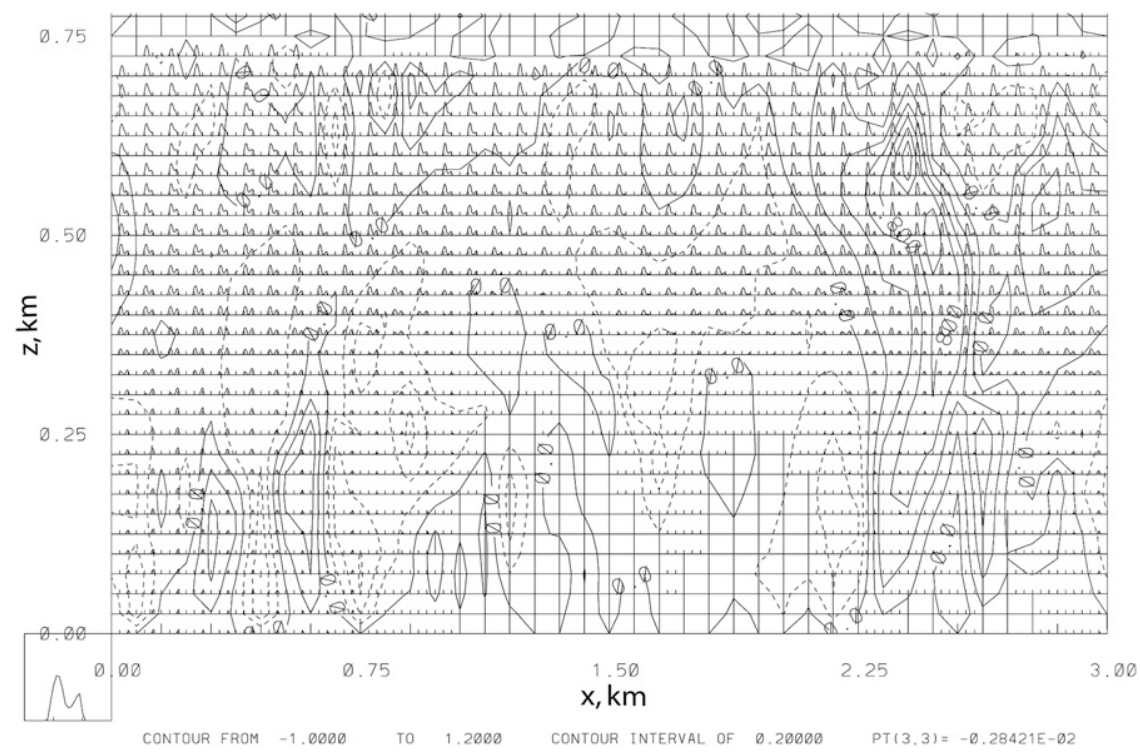

FIG. 2. Cloud droplet spectra in the MD case superimposed with isolines of the vertical velocity $\left(\mathrm{m} \mathrm{s}^{-1}\right)$ in a vertical cross section through the cloud layer. At each spatial point, the square box representing the drop mass distribution function vs $\log r$ is shown. The large box in the lower left corner shown for illustration has two tick marks at the bottom corresponding to $10-$ and $100-\mu \mathrm{m}$ drop radius.

where parameters are $N, \alpha$, and $\beta$. The gamma function $\Gamma(x)$ is defined as

$$
\Gamma(x)=\int_{0}^{\infty} t^{x-1} e^{-t} d t
$$

Moments of the DSD (5) are given by the expression (see, e.g., Frisch et al. 1998)

$$
M_{k}=\int_{r=0}^{\infty} r^{k} n(r) d r=N \beta^{k} \frac{\Gamma(\alpha+k+1)}{\Gamma(\alpha+1)} .
$$

To obtain the three parameters defining analytical fit (2) or (5), we first calculate the zero, first, and second moments of the LES-derived DSDs. Substituting the values of these moments into (3) or (7) for $k=0,1,2$ yields three algebraic equations for parameters $N, \sigma_{\ln r}$, and $r_{m}$ in the case of lognormal distribution, or for parameters $N, \alpha, \beta$ in the case of gamma distribution (see the appendix). In principle, any three moments yield solutions for the analytic DSD parameters; the specific choice of $k=0,1$, and 2 corresponds to the typical notion of specifying a distribution by mean, variance, and total number $(k=0)$. The fidelity of the analytic DSDs are evaluated by comparing the fourth and sixth moments of each fit with the corresponding moments of the DSD calculated from the LES dataset. Note that in boundary layer stratocumuli, these moments are proportional to the drizzle flux and radar reflectivity.
Depending on drizzle intensity, drop spectra in stratocumulus may exhibit one or two modes: the first mode represents cloud $(r<25 \mu \mathrm{m})$ and the second mode represents drizzle drops $(r \geq 25 \mu \mathrm{m})$ (see, e.g., Figure 2). We, therefore, consider two fit types. The unimodal fit is defined by three parameters expressed through moments of the DSD integrated over the whole drop size range. The unimodal fit obviously is suitable for approximating the DSD in nonprecipitating clouds, whereas a bimodal representation will clearly be more accurate in precipitating clouds. The bimodal fit is a sum of two analytical functions, defined by three parameters expressed through moments integrated over the 1) cloud drop sizes, and 2) drizzle drop sizes.

\section{Approximation of drop size distributions in marine stratocumulus}

\section{a. Evaluating three-parameter lognormal and gamma distributions}

As mentioned above, the DSDs in light drizzling stratocumulus are mostly unimodal. The results shown in Fig. 3 demonstrate that in this case, the DSDs can be accurately approximated even by unimodal analytical fits. The accuracy of the approximation is progressively improved when a gamma distribution is employed in place of a lognormal function (Fig. 3, middle), and when the two-mode fit is used instead of a single mode fit 

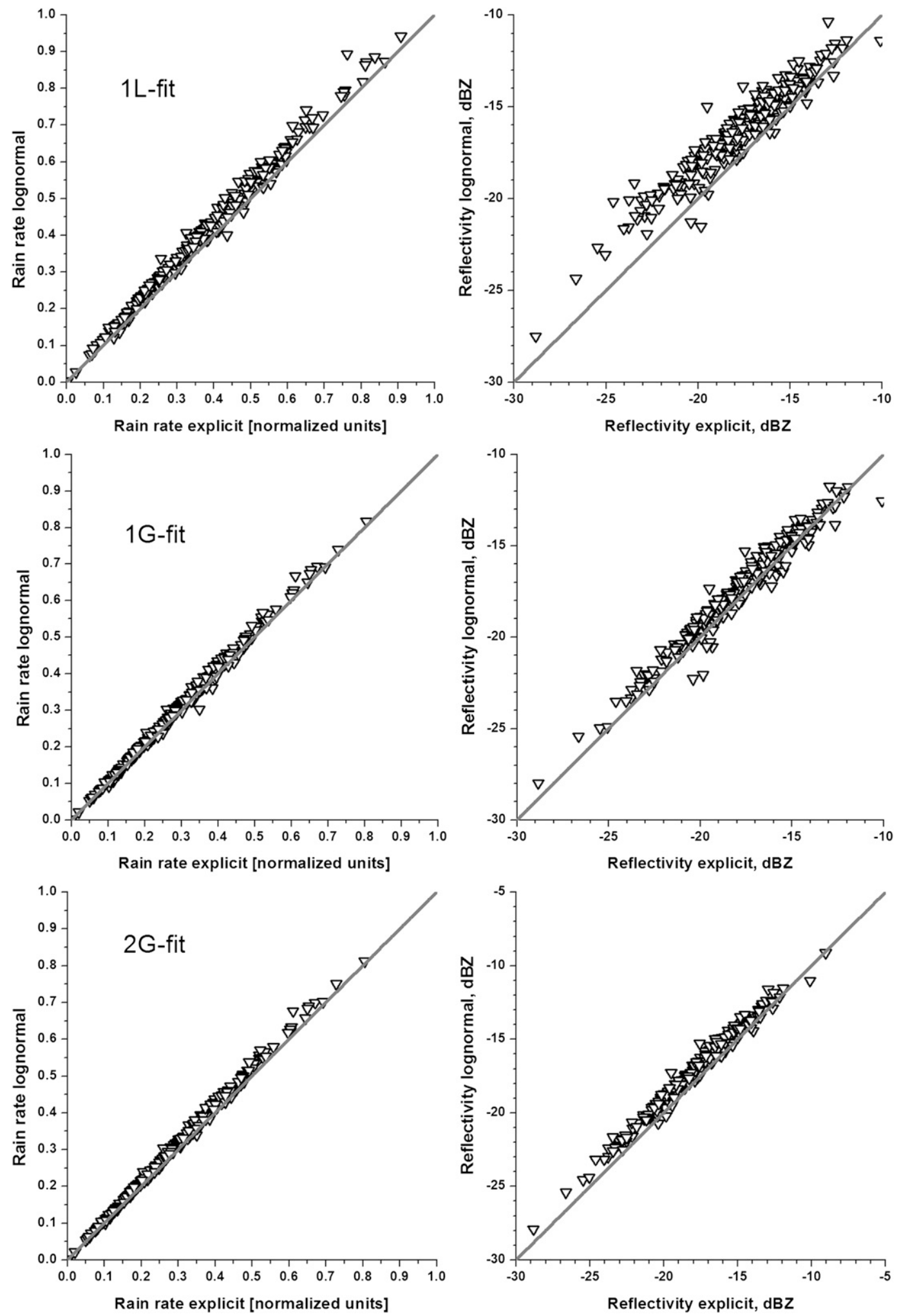

FIG. 3. Scatterplots of rain rate and radar reflectivity in the LD case approximated by analytical fits vs exact parameters calculated from the explicit microphysics model simulations: (top) unimodal lognormal fit (1L-fit); (middle) unimodal gamma fit (1G-fit), and (bottom) bimodal G-fit. 

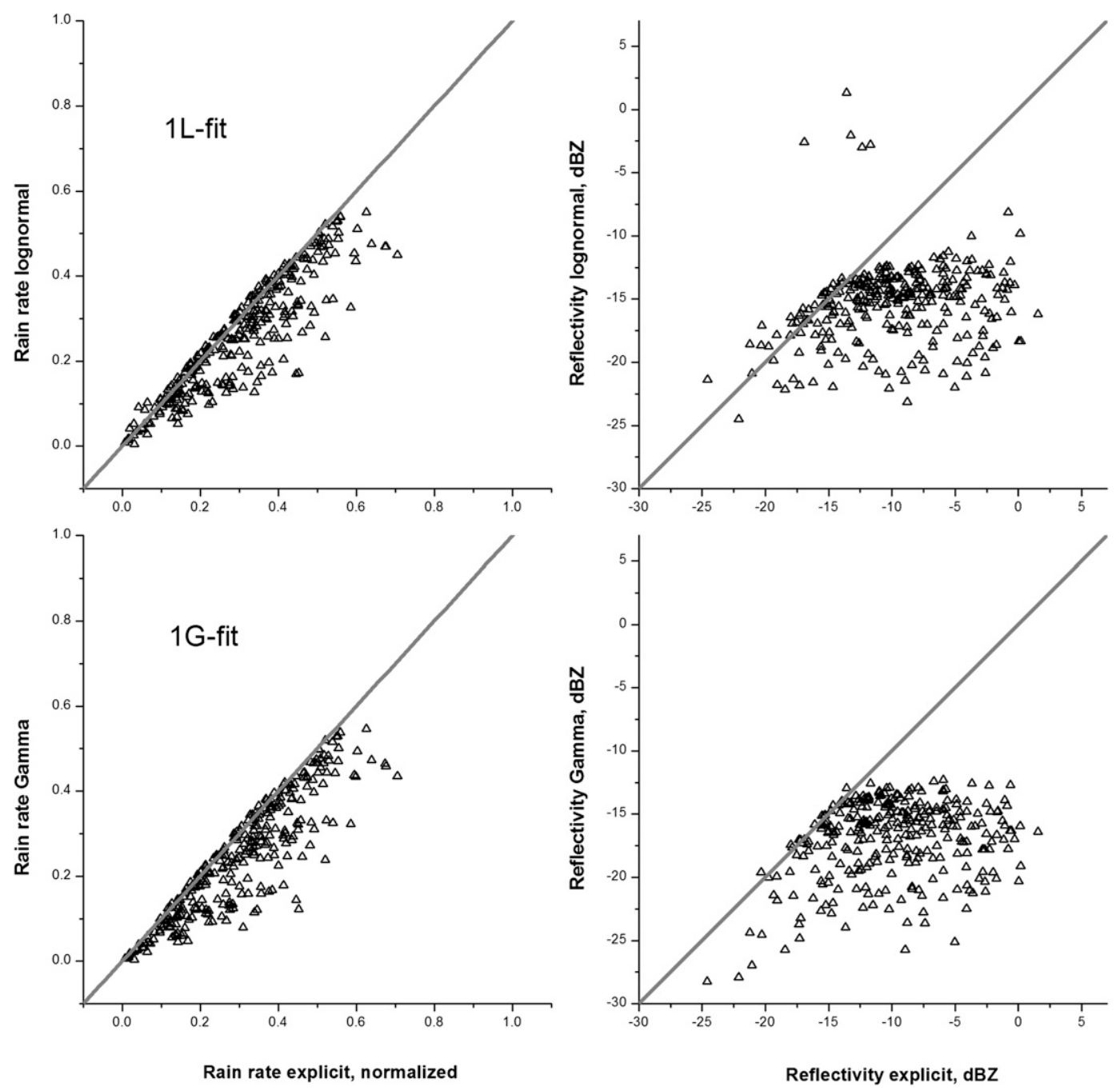

FIG. 4. Rain rate and radar reflectivity in the MD case as approximated by unimodal fits.

(Fig. 3, bottom). Figure 3 demonstrates that the rain rate is approximated more accurately than radar reflectivity, obviously because the higher moments have an increased contribution from large droplets in the tail of the spectrum. The analytical approximations tend to overestimate radar reflectivity, particularly when singlemode L-fits are employed. Nevertheless, both analytical fits work reasonably well in this light drizzle case. In the case of nondrizzling clouds (not shown), both analytical fits provide highly accurate approximation of the DSDs.

Rain rate and radar reflectivity in the MD case are significantly underestimated by either an L-fit (Fig. 4, left), or a G-fit (Fig. 4, right) because the unimodal distributions fail to capture the contribution from the tail of the spectra. This underestimation is significantly reduced when bimodal fits are used (Fig. 5). The comparison between Figs. 4 and 5 also reveals that the bi- modal fits yield a smaller bias relative to the unimodal fits. The bimodal G-fits also have a substantially reduced scatter and less bias, especially for evaluating rain rates. The same conclusions hold for the HD case (Fig. 6). Comparison of error envelopes (mean \pm standard deviation) between L-fits and G-fits for the HD case is shown in Fig. 7. The errors of approximation by L-fits are much larger, especially for the small and middle ranges of rain rates and reflectivities.

\section{b. Defining analytical fits in models with two-moment cloud parameterizations}

In the previous section, the drop size distributions were approximated by three-parameter analytical fits. Some current mesoscale numerical prediction models (M. Xue 2008, personal communication) already incorporate three-moment cloud physics parameterizations 

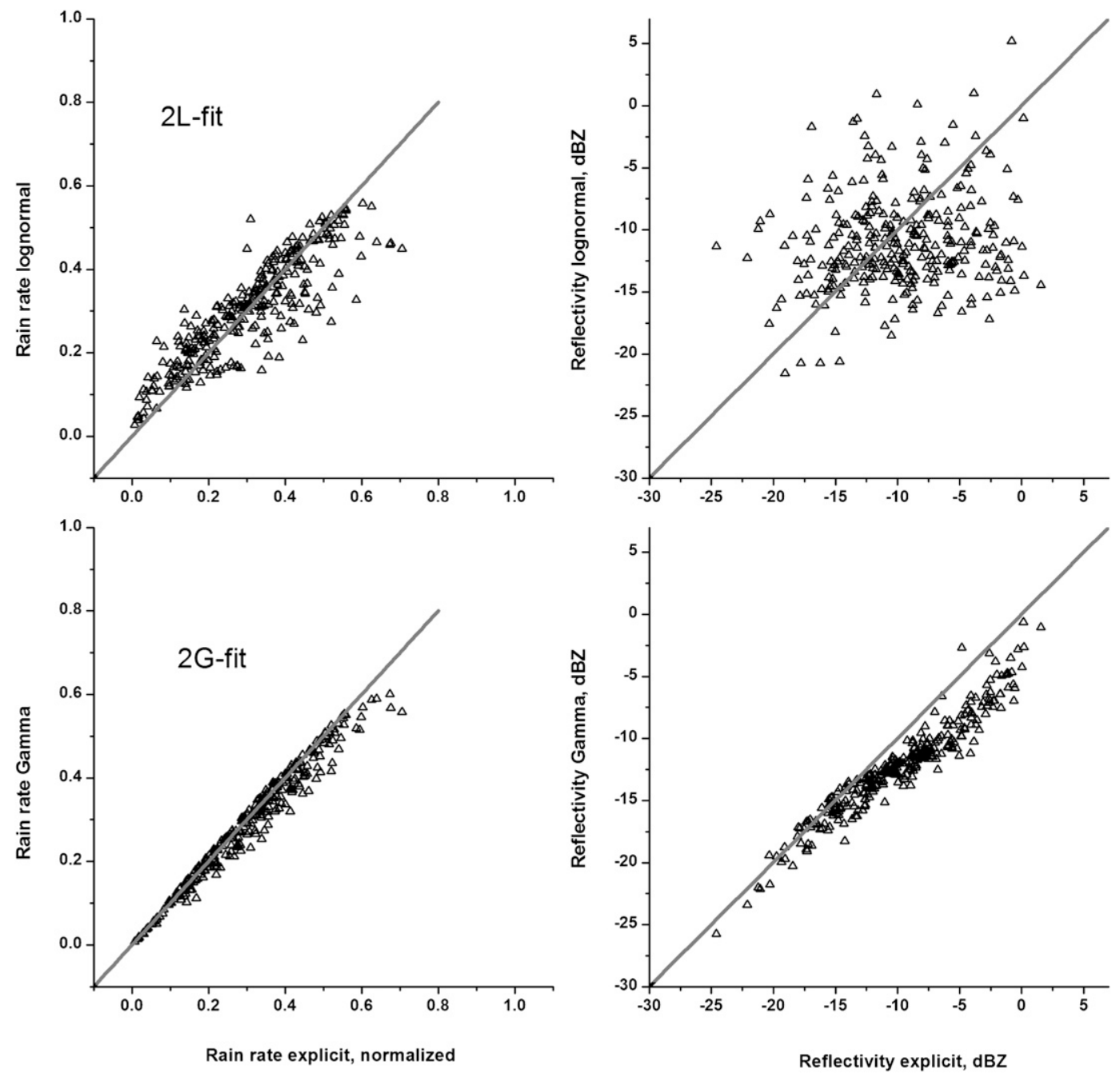

FIG. 5. As in Fig. 4, but for bimodal fits.

(Milbrandt and Yau 2005a,b) and thus are capable of relating moments to the three parameters of an analytical distribution. On the other hand, the majority of state-of-the-art cloud and mesoscale prediction models still use two-moment schemes, meaning that only two prognostic variables are available for defining the analytical DSDs.

In this section we describe an approach to reduce the number of parameters defining a DSD analytical fit in marine stratocumulus clouds from three to two. This is accomplished by formulating a simple closure for the dispersion as a function of two other parameters of the DSD: drop concentration and mixing ratio. Drop concentration and mixing ratio are typically the prognostic microphysical variables in two-moment cloud parameterizations, both for cloud and drizzle drops.

The relative dispersion of the drop spectra is by definition related to the moments of the LES-derived DSDs,

$$
\sigma^{2}=\frac{\overline{m_{2}}}{{\overline{m_{1}}}^{2}}-1
$$

where $\bar{m}_{1}$ and $\bar{m}_{2}$ represent the first and second moments normalized by drop concentration $N$.

Figure 8 shows that the dispersion of the cloud drop size distribution $\sigma_{c}$ is a decreasing function of $N_{c}$. For $N_{c}<30 \mathrm{~cm}^{-3}$, a range that corresponds to large drizzle rates, the dispersion can be roughly approximated by a linear function,

$$
\sigma_{c}=0.54-0.0094 N_{c} .
$$

For $N_{c}>30 \mathrm{~cm}^{-3}, \sigma_{c}$ can be approximated by a constant value of 0.25 . Since our focus is on drizzling stratocumulus, the relationship (9) is applicable only to the range of droplet concentrations corresponding to stratocumulus evolving under very clean maritime conditions. 

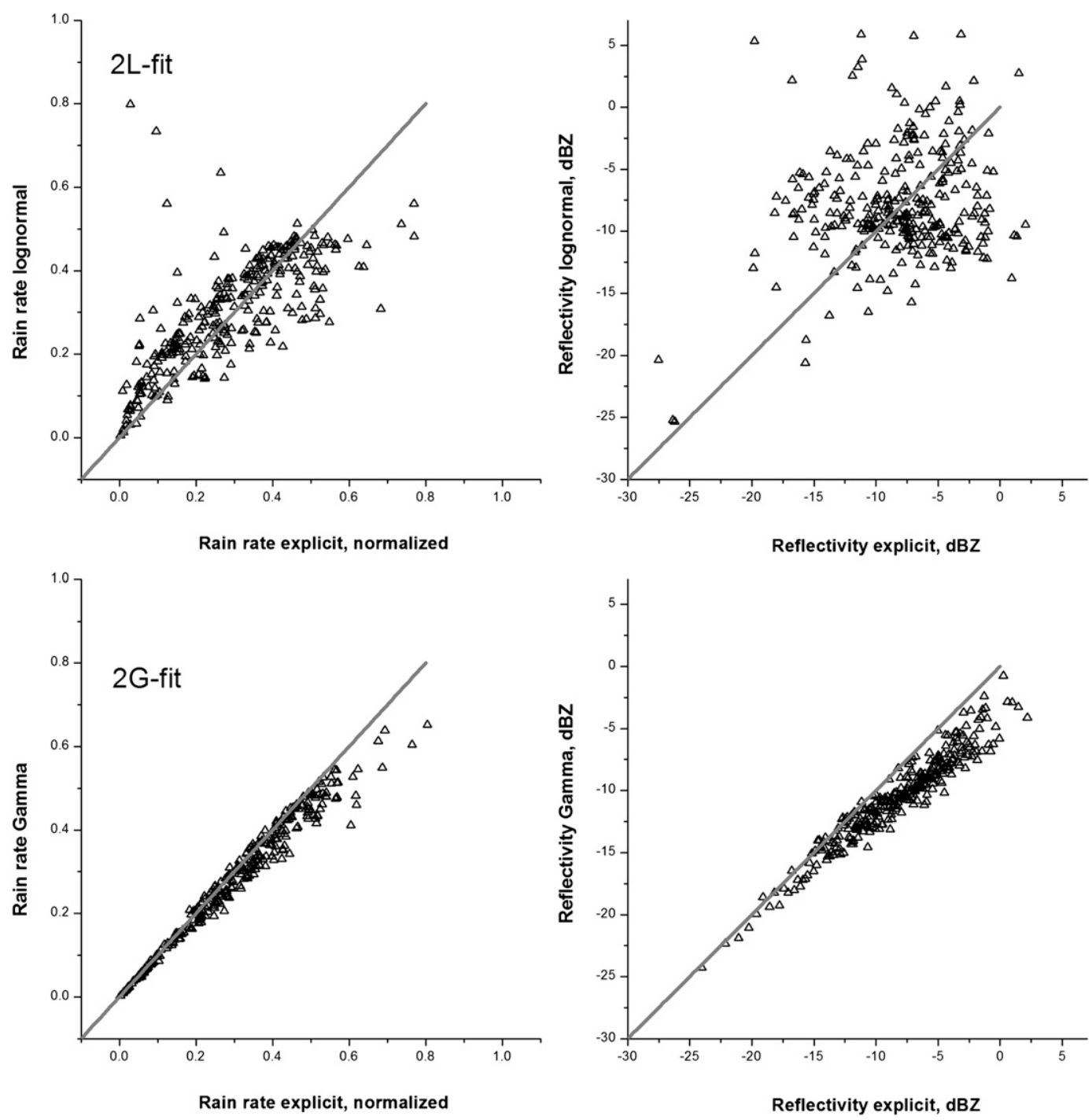

FIG. 6. As in Fig. 5, but for the HD case.

For much larger values of $N_{c}$ characterizing nonprecipitating clouds, observations reveal a more complex relationship between $\sigma_{c}$ and $N_{c}$ (see, e.g., Pawlowska et al. 2006). For the precipitating stratocumulus in our cases, the cloud drops contribute only minimally to the higher-order moments, and the ultimate choice of $\sigma_{c}$ [either Eq. (9) or assuming a constant value] has a very small effect on the results.

The dispersion $\sigma_{d}$ of the drizzle drop spectra $(r \geq 25$ $\mu \mathrm{m})$ is shown in Fig. 9. We first tested a fit for $\sigma_{d}$ based on a linear function of drizzle drop concentration $N_{d}$ :

$$
\sigma_{d}=a-b N_{d}
$$

where $a=0.38$ and $b=-0.14 \mathrm{~cm}^{3}$.

The solid gray line in Fig. 9 represents this linear fit. The same figure shows that the dependence of $\sigma_{d}$ on drizzle drop concentration $N_{d}$ is more meaningful when the DSDs are stratified by drizzle drop mixing ratio $Q_{d}$. In this case, the coefficients $a$ and $b$ are not constant but rather are expressed as a quadratic function of drizzle drop mixing ratio:

$$
\begin{aligned}
& a=0.33+3.76 Q_{d}-13.5 Q_{d}^{2} \\
& b=-1.02+8.63 Q_{d}-22.9 Q_{d}^{2} .
\end{aligned}
$$

Here, $Q_{d}$ varies in the range from 0 to $0.24 \mathrm{~g} \mathrm{~m}^{-3}$.

Figure 10 compares the results of using the G-fit, employing the closure for $\sigma_{d}$ specified by (10) and (11). Obviously, calculating rain rates and reflectivity (top) from all three parameters is the most accurate approach. When the G-fit is determined by only two parameters 

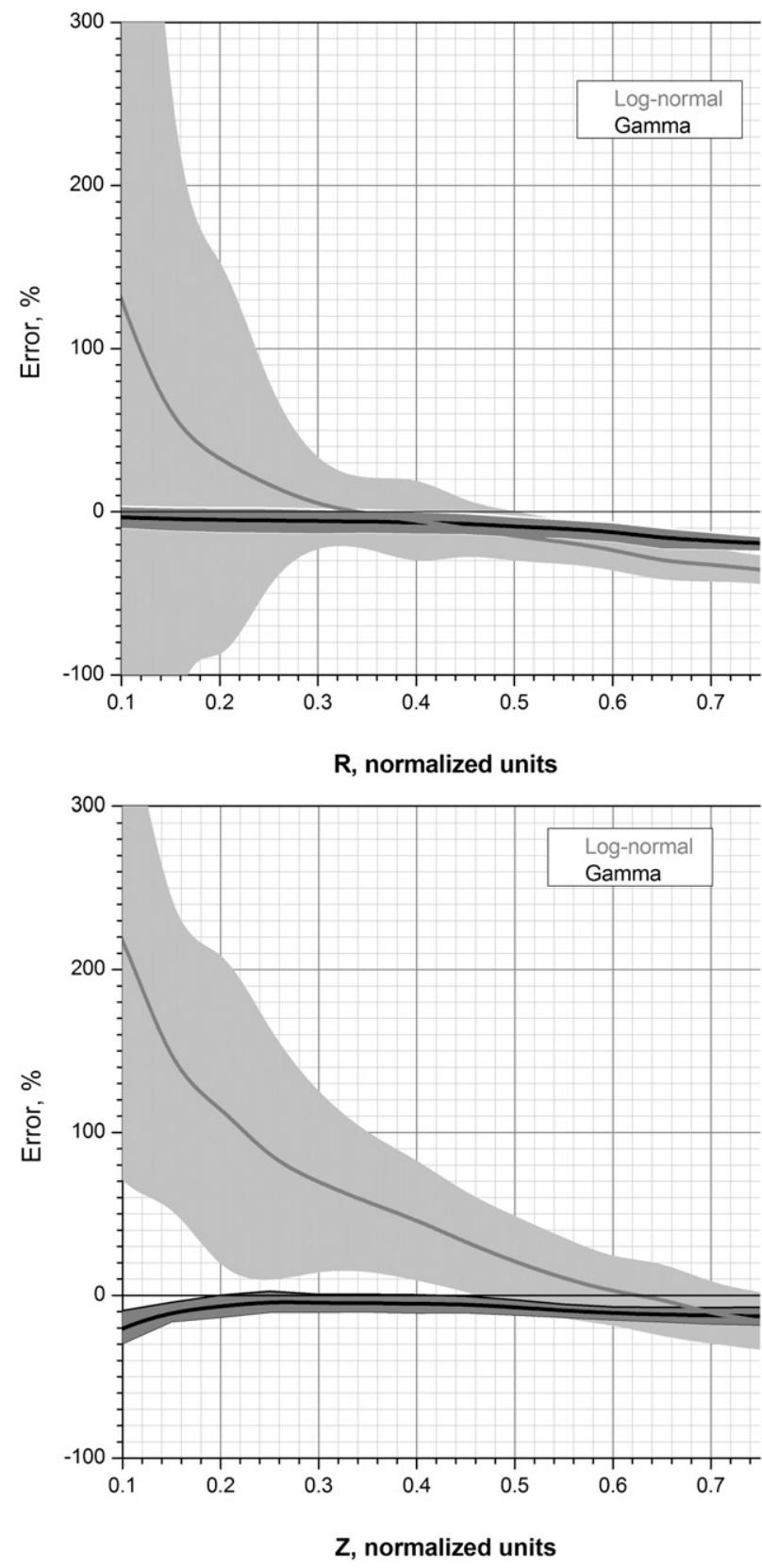

FIG. 7. Errors of approximation of $R$ and $Z$ from bimodal analytical fits in the HD case. Radar reflectivity range $(-35,+5 \mathrm{~dB} Z)$ was linearly transformed into the $(0,1)$ interval.

with dispersion expressed by (10), the accuracy is significantly reduced (middle), resulting in more scatter for precipitation flux and increased bias for reflectivity. However, representing the dispersion by (11) yields a reduction of scatter for precipitation flux and less bias for reflectivity, although the scatter in the latter case is still significant, especially for large reflectivities (bottom).

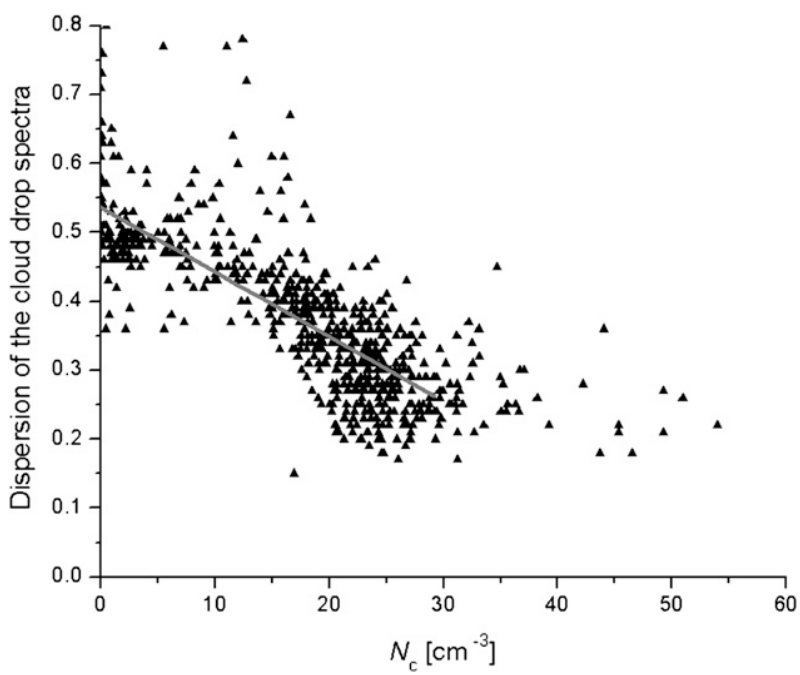

FIG. 8. Dispersion of the cloud drop size distribution as a function of cloud drop concentration in the HD case. The linear fit is shown by the gray line.

\section{c. Formulation of radar reflectivity in bulk models}

Radar reflectivity $Z$ is not explicitly predicted in bulk models. Its knowledge, nevertheless, is important for comparing model predictions with direct radar observations. The question is how to derive values of radar reflectivity based on the model prognostic variables. In twomoment microphysical schemes, these variables include concentrations and mixing ratios for the cloud droplet and drizzle categories, namely, $N_{c}, Q_{c}, N_{d}$, and $Q_{d}$.

Figure 11 shows the fidelity of the model-derived reflectivity fields by contrasting different approximations for DSDs with the case in which $Z$ is calculated as a sixth moment of the full DSD predicted by the explicit microphysics LES model (Fig. 11a). The same field using

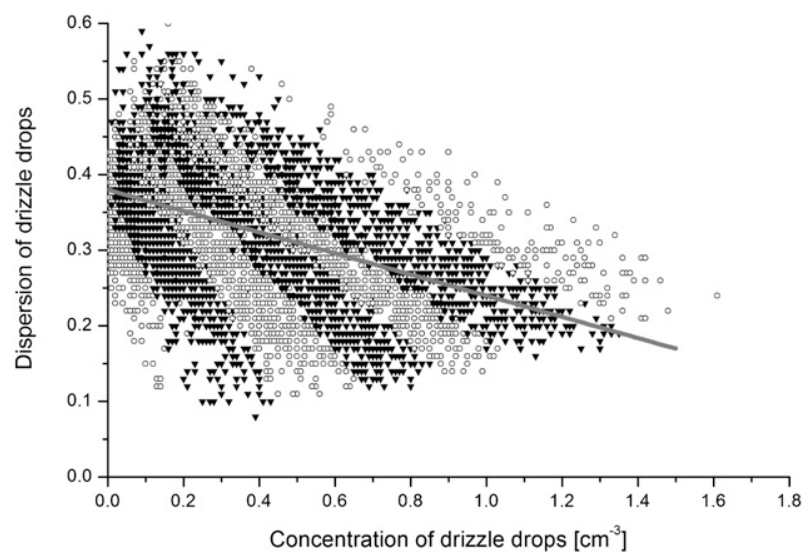

FIG. 9. Scatterplots of the drizzle drops dispersion as a function of drizzle drop concentration. The data points are stratified by the value of $Q_{d}$ in the range from 0.02 to $0.24 \mathrm{~g} \mathrm{~m}^{-3}$. (left)-(right) Each change in symbol corresponds to the increase in $Q_{d}$ by $0.04 \mathrm{~g} \mathrm{~m}^{-3}$. 

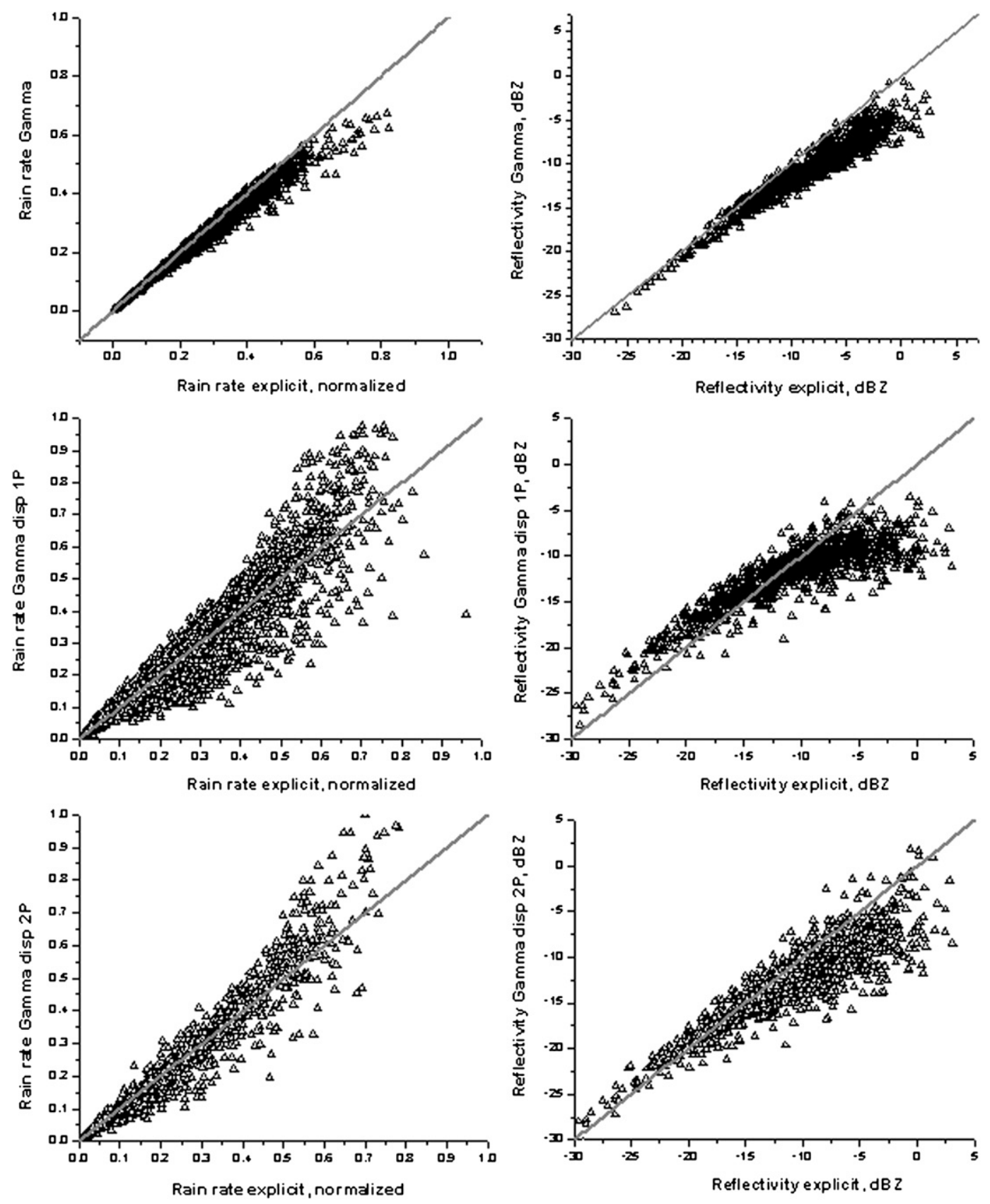

FIG. 10. The scatterplots of rain rates and reflectivity approximated by gamma fits in the HD case. (top) The performance of the original three-parameter gamma fit. (middle) The two-parameter gamma fit with the $\sigma=f\left(N_{d}\right)$. (bottom) The case in which $\sigma=f\left(N_{d}, Q_{d}\right)$. Rain rate was normalized by dividing by the maximum rain rate in the dataset.

the DSDs approximated by the two-mode three-parameter gamma function is shown in Fig. 11b. The three-parameter G-fit correctly shows the general structure of the $Z$ field, with the exception of one area below the cloud top. This is the region where, because of early coagulation, the cloud and drizzle modes in the DSD overlap and are not distinctly separated. The three-parameter G-fit is more ac- curate in areas near cloud base. Here, because of the further coagulation of drops and consequent sedimentation, the two modes overlap less, leading to more accurate three-parameter G-fits.

Figure 11c shows the $Z$ field for the case in which DSDs are fitted with the two-parameter gamma distribution, where $\sigma$ is calculated according to (9), (10), and 

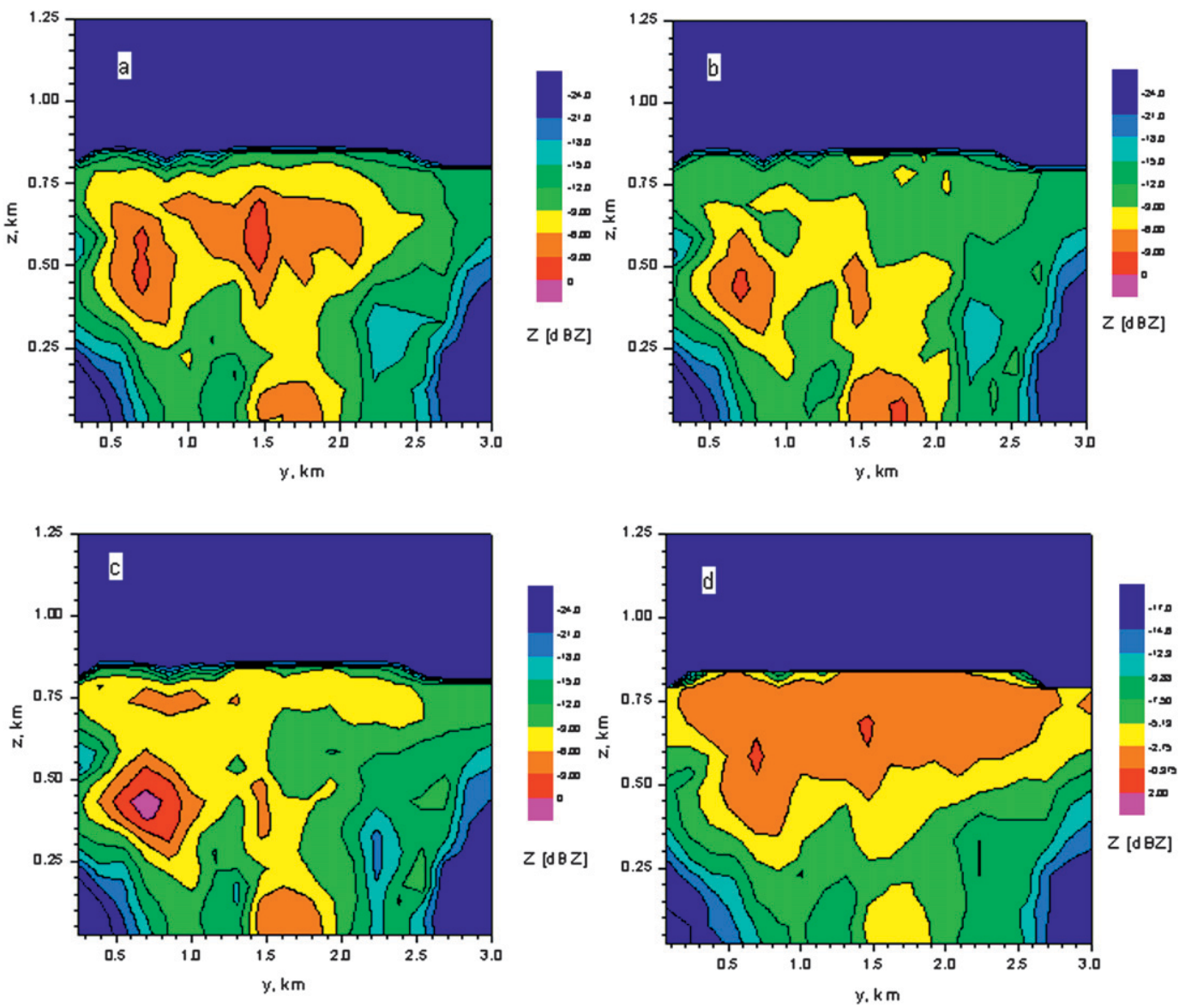

FIG. 11. The vertical cross section of radar reflectivity field in four cases: (a) $Z$ calculated from the full LES predicted DSDs; (b) $Z$ defined from the three-parameter gamma fit; (c) $Z$ defined by the two-parameter gamma fit with $\sigma$ calculated according to (9) and (11); and (d) $Z$ defined from the $Z-R$ relationship.

(11). In this case the resulting reflectivity field is very similar to the field employing the three-parameter G-fits; however, the area of high reflectivities near cloud top is missed in this case as well.

To assess the advantages and limitations of the twomode gamma approximation, we contrast it in Fig. 11d with the reflectivity field calculated from the $Z-R$ relationship. The latter is given by the following expression:

$$
Z=a R^{b}
$$

where $R$ is the precipitation flux, and parameters $a$ and $b$ are constants calculated from the explicit microphysics model DSD dataset. Tests of the HD case show that the best correlation between the reflectivity calculated directly from the complete droplet spectra and the reflectivity derived using (12) based on the precipitation flux obtained from the explicit model was achieved using $a=2.6$ and $b=0.6$. These best-fit parameters are similar to those found by vanZanten et al. (2005), based on observations of stratocumulus off the California coast during the Second Dynamics and Chemistry of Marine Stratocumulus field study (DYCOMS II). Their analysis of the in situ particle probe data yielded values in the range of 1.66-2.7 and $0.46-0.74$ for $a$ and $b$, respectively (Table 2 in vanZanten et al. 2005). ${ }^{2}$

The most apparent limitation in the $Z-R$ relationship lies in its poor representation of the finescale reflectivity

\footnotetext{
${ }^{2}$ The parameters $a$ and $b$ in stratocumulus can vary significantly. For example, from observations of stratus off the coast of Oregon, Vali et al. (1998) suggested the value of parameter $b=1.0$. Comstock et al. (2004), from observations of southeastern Pacific stratocumulus, concluded that parameter $b$ is in the range 1.1-1.4. We note the significant differences in environmental conditions and cloud properties between the northwestern and southeastern Pacific, as contrasted between the DYCOMS II (Stevens et al. 2003) and East Pacific Investigation of Climate Processes in the Coupled Ocean-Atmosphere System (EPIC; Bretherton et al. 2004) field projects. Obviously these parameters are highly sensitive to environmental conditions defining stratocumulus clouds.
} 
structures evident in Fig. 11d, whereas these structures are captured more accurately using the three- and twoparameter gamma approximations. It should be noted that parameters $a$ and $b$ in the employed $Z-R$ relationship were calculated from the complete DSD spectra corresponding to a specific case of drizzling stratocumulus. Unfortunately, this type of case-by-case tuning of the parameters is not possible in bulk models, where information on DSD is not available. As for any $Z-R$ relationship, the generality of our parameters $a$ and $b$ is limited and should not be thoughtlessly applied to other cases because the application of $Z-R$ parameters in conditions for which they were not formulated can introduce significant error into the calculated reflectivity.

\section{Discussion}

Cloud microphysical parameterizations and retrievals rely to a significant degree on the knowledge of the shape of drop size distributions (DSDs). Many investigations assume that DSDs, either in whole or in part, may be approximated by known analytical functions. This study evaluates the fidelity of two frequently used analytical fits (gamma and lognormal) employed for approximating DSDs in boundary layer stratocumulus by evaluating how well they represent the higher-order moments (fourth and sixth) of the DSD. The higherorder moments from the analytical fits are contrasted with the moments calculated from DSDs obtained from simulations of drizzling marine stratocumulus clouds observed during ASTEX. The numerical experiments were made using the CIMMS large-eddy simulation model with size-resolving microphysics. Three simulations represent different intensities of in-cloud drizzle: light (LD), moderate (MD) and heavy (HD) drizzle. Each of these simulations provided more than 19000 DSDs for a wide range of drizzling conditions. The DSDs were used to define the parameters of the analytical fits and to serve as a benchmark for evaluating the fidelity of the DSD approximations.

We note that, depending on drizzle intensity, drop spectra in stratocumulus may exhibit one or two modes, with the first mode representing cloud $(r<25 \mu \mathrm{m})$ and the second mode representing drizzle drops $(r \geq 25$ $\mu \mathrm{m})$. We, therefore, consider two types of analytical fits: unimodal and bimodal.

The unimodal fit is defined by three parameters expressed through moments of the DSD integrated over the whole drop size range. The unimodal fits can be appropriate for approximating the DSD in nonprecipitating or lightly drizzling clouds. In clouds where precipitation is well developed, one can expect the bimodal fits to be more accurate. Bimodal distributions are defined here as the sum of two fits, the first defined by three parameters expressed through partial moments integrated over the cloud droplet sizes and the second defined by three parameters expressed through moments integrated over the drizzle drop sizes. We found that unimodal fits can represent rain rates and radar reflectivity rather well for nonprecipitating or slightly precipitating conditions. Under these conditions, the gamma distribution appears to provide a more accurate estimate of the rain rates and radar reflectivity. In cloud layers with heavier drizzle (more than $1 \mathrm{~mm} \mathrm{~d}^{-1}$ ), bimodal fits are significantly more accurate than unimodal. The gammatype distributions fitted for each mode represent rain rates and radar reflectivities much more accurately than lognormal fits, particularly for the low-to-midrange of rain rates and reflectivities.

Our results, thus, show that drop size distributions in boundary layer marine stratocumulus can be most accurately approximated by six parameters defining the two-mode gamma-type function. Some current cloud resolving and mesoscale models include sophisticated threemoment cloud physics parameterizations (Milbrandt and Yau 2005a,b) and, consequently, predict six variables that can be related to cloud and drizzle concentrations, mixing ratios, and drop spectra dispersions. These six predictive variables can be straightforwardly linked to the six parameters of the two-mode analytical gamma or lognormal functions. Models incorporating this level of microphysical sophistication are, therefore, best capable of representing both rain rates and radar reflectivity, constituting an improvement in accuracy relative to simpler one- and two-moment parameterizations.

However, the majority of state-of-the-art cloudresolving and mesoscale models still use the two-moment cloud microphysics formulation. In such models only four prognostic variables are available for constraining the analytical DSDs. Our results have demonstrated that one of the defining parameters of the DSD spectrum dispersion - can be approximated as a function of the two other parameters of the DSD: drop concentration and mixing ratio. The latter parameters are predicted in two-moment cloud parameterizations, separately for cloud and drizzle drops. In the two-moment schemes, the dispersion of the drizzle drop distribution can be expressed as a linear function of the drizzle drop concentration, with parameters of the linear fit expressed by quadratic functions of the drizzle drop mixing ratio.

Note that a dispersion closure for conditions characteristic of convective clouds with heavier precipitation was proposed by Milbrandt and Yau (2005a), who demonstrated the importance of dispersion for obtaining an accurate estimation of sedimentation rates. Morrison 
et al. (2005) proposed a new double-moment bulk parameterization of cloud physics processes that incorporated the expression for droplet dispersion developed by Khvorostyanov and Curry (1999). It is notable that these parameterization developments were based on the use of the gamma-type distributions for drop spectra as well.

In summary, for precipitating boundary layer marine stratocumulus, our results indicate that an analytic DSD based on the two-mode gamma distribution is more accurate than the DSD based on the lognormal distribution. The gamma distribution also provides a more accurate representation of the radar reflectivity field in two- and three-moment bulk microphysical models compared to the conventional $Z-R$ relationship.

Acknowledgments. This research was supported by ONR Grants N00014-03-1-0304, N00014- 05-1-0550, and N00014-08-1-0681; by the Office of Science (BER), U.S. Department of Energy Grant DE-FG02-05ER64062; and by NOAA's Office of Oceanic and Atmospheric Research under NOAA-University of Oklahoma (OU) Cooperative Agreement NA17RJ1227, U.S. Department of Commerce. Some of the computing for this project was performed at the OU Supercomputing Center for Education \& Research (OSCER) at The University of Oklahoma. We appreciate the constructive comments of anonymous reviewers that led to the improvement of this manuscript.

\section{APPENDIX}

\section{Calculation of Gamma Function Parameters}

The following expressions follow from the definition of moments of the gamma function (7):

$$
\begin{gathered}
M_{k} / M_{k-1}=\beta(\alpha+k) \quad \text { and } \\
M_{k-1} / M_{k-2}=\beta(\alpha+k-1) .
\end{gathered}
$$

Subtracting (A2) from (A1) provides the value of $\beta$ :

$$
\beta=M_{k} / M_{k-1}-M_{k-1} / M_{k-2} .
$$

Dividing (A1) by (A2) results in a simple linear equation for $\alpha$ :

$$
\alpha=M_{k} M_{k-2} / M_{k-1}^{2}-k+1 .
$$

The normalization factor $N$ then follows from definition (7):

$$
M_{k}=N \beta^{k} \frac{\Gamma(\alpha+k+1)}{\Gamma(\alpha+1)} .
$$

Once $N, \alpha, \beta$ are known, moments for any index $k$ can be obtained from (A5). In this study we used $k=2$ for calculating parameters of the gamma distribution and then used (A5) for calculating the fourth and sixth moments. However, (A1)-(A5) hold for any value of $k$. Similar simple math manipulations allow calculations of parameters of the lognormal distribution.

\section{REFERENCES}

Albrecht, B. A., C. S. Bretherton, D. Johnson, W. H. Shubert, and A. S. Frisch, 1995: The Atlantic stratocumulus transition experiment-ASTEX. Bull. Amer. Meteor. Soc., 76, 889-904.

Bretherton, C. S., and Coauthors, 2004: The EPIC 2001 stratocumulus study. Bull. Amer. Meteor. Soc., 85, 967-977.

Cerro, C., B. Codina, J. Bech, and J. Lorente, 1997: Modeling raindrop size distribution and $Z(R)$ relations in the western Mediterranean area. J. Appl. Meteor., 36, 1470-1479.

Comstock, K., R. Wood, S. E. Yuter, and C. S. Bretherton, 2004: Reflectivity and rain rate in and below drizzling stratocumulus. Quart. J. Roy. Meteor. Soc., 130, 2891-2918.

Feingold, G., and Z. Levin, 1986: The lognormal fit to raindrop spectra from frontal convective clouds in Israel. J. Climate Appl. Meteor., 25, 1346-1363.

Frisch, A. S., G. Feingold, C. W. Fairall, T. Uttal, and J. B. Snider, 1998: On cloud radar and microwave radiometer measurements of stratus cloud liquid water profiles. J. Geophys. Res., 103, 23 195-23 197.

Gerber, H., 1996: Microphysics of marine stratocumulus clouds with two drizzle modes. J. Atmos. Sci., 53, 1649-1662.

Khairoutdinov, M. F., and Y. L. Kogan, 1999: A large eddy simulation model with explicit microphysics: Validation against aircraft observations of a stratocumulus-topped boundary layer. J. Atmos. Sci., 56, 2115-2131.

Khvorostyanov, V. I., and J. A. Curry, 1999: Toward the theory of stochastic condensation in clouds. Part II: Analytical solutions of the gamma-distribution type. J. Atmos. Sci., 56, 3997-4013.

Kogan, Y. L., 1991: The simulation of a convective cloud in a 3-D model with explicit microphysics. Part I: Model description and sensitivity experiments. J. Atmos. Sci., 48, 1160-1189.

—, M. P. Khairoutdinov, D. K. Lilly, Z. N. Kogan, and Q. Liu, 1995: Modeling of stratocumulus cloud layers in a large eddy simulation model with explicit microphysics. J. Atmos. Sci., 52, 2923-2940.

Liu, Q., Y. L. Kogan, D. K. Lilly, D. W. Johnson, G. E. Innis, P. A. Durkee, and K. Nielson, 2000: Modeling of ship effluent transport and its sensitivity to boundary layer structure. J. Atmos. Sci., 57, 2779-2791.

Milbrandt, J. A., and M. K. Yau, 2005a: A multimoment bulk microphysics parameterization. Part I: Analysis of the role of the spectral shape parameter. J. Atmos. Sci., 62, 3051-3064.

- , and $-2005 \mathrm{~b}$ : A multimoment bulk microphysics parameterization. Part II: A proposed three-moment closure and scheme description. J. Atmos. Sci., 62, 3065-3081.

Morrison, H., J. A. Curry, and V. I. Khvorostyanov, 2005: A new double-moment microphysics parameterization for application in cloud and climate models. Part I: Description. J. Atmos. Sci., 62, 1665-1677.

O'Connor, E. J., R. J. Hogan, and A. J. Illingworth, 2005: Retrieving stratocumulus drizzle parameters using Doppler radar and lidar. J. Appl. Meteor., 44, 14-27. 
Pawlowska, H., W. W. Grabowski, and J.-L. Brenguier, Observations of the width of cloud droplet spectra in stratocumulus. Geophys. Res. Lett., 33, L19810, doi:10.1029/ 2006GL026841.

Stevens, B., and Coauthors, 2003: Dynamics and chemistry of marine stratocumulus-DYCOMS-II. Bull. Amer. Meteor. Soc., 84, 579-593.

Vali, G., R. D. Kelly, J. French, S. Haimov, D. Leon, R. E. McIntosh, and A. Pazmany, 1998: Finescale structure and microphysics of coastal stratus. J. Atmos. Sci., 55, 3540-3564.
vanZanten, M. C., B. Stevens, G. Vali, and D. H. Lenschow, 2005: Observations of drizzle in nocturnal marine stratocumulus. J. Atmos. Sci., 62, 88-106.

Willis, P. T., 1984: Functional fits to some observed drop size distributions and parameterization of rain. J. Atmos. Sci., 41, 1648-1661.

Wood, R., 2005: Drizzle in stratiform boundary layer clouds. Part II: Microphysical aspects. J. Atmos. Sci., 62, 3034-3050.

, P. R. Field, and W. R. Cotton, 2002: Autoconversion rate bias in stratiform boundary layer cloud parameterizations. Atmos. Res., 65, 109-128. 